\title{
A TRP channel-steroid marriage
}

\author{
Bernd Nilius and Thomas Voets
}

A surprising functional association between TRPM3, a mysterious member of the family of transient receptor potential
(TRP) cation channels, and the sulphated version of pregnenolone, 'mother' of all steroid hormones, has been identified.

The TRP family (Fig. 1a) carries the stigma of being 'promiscuous', as its members can be tempted to gate in response to a surprising variety of stimuli, including temperature and mechanical stress, as well as various endogenous and exogenous chemicals. The steroid hormone pregnenolone and its water-soluble ester pregnenolone sulphate (PS) seem to be even more variable. Pregnenolone, which is derived from cholesterol, is the precursor of other steroids, including progesterone, mineralocorticoids, glucocorticoids, androgens and oestrogens, and can be sulphonated to form pregnenolone sulphate (PS; Fig. 1b). During the 1940s, pregnenolone was reported to have anti-stress and mood-elevating effects on factory workers, students and pilots. Later studies demonstrated that pregnelonone and PS act as neurosteroids, causing improved synaptic functioning, neuroprotection, enhanced myelinization and increased hippocampal neurogenesis. In addition to improving cognitive functions and memory, pregnenolone also enhances stage IV sleep, and it may have anti-ageing and anti-arthritic effects ${ }^{1-4}$, thus being billed as a 'fountain of youth' (Fig. 1c).

How can pregnenolone and PS possibly accomplish all of these effects? Classically, the physiological actions of steroid hormones have been attributed to nuclear steroid receptors, which directly influence gene expression upon binding of the steroid ligand. However, there is increasing evidence for faster, non-genomic effects of steroids, through a direct interaction

Bernd Nilius and Thomas Voets are in the Department of Molecular Cell Biology, Laboratory of Ion Channel Research, KU Leuven, Campus Gasthuisberg, Herestraat 49, bus 802, B-3000 Leuven, Belgium.

e-mail: bernd.nilius@med.kuleuven.be with membrane receptors. For example, in the case of PS, modulatory effects on $\mathrm{GABA}_{\mathrm{A}}{ }^{5,6}$, glutamate NR1/NR2 ${ }^{7}$ and nicotinic acetylcholine receptors ${ }^{8}$ have been described. On page 1421 of this issue, Wagner et al. show that a TRP channel (TRPM3) is directly activated by PS 9

Until now, TRPM3 was, in many respects, a mysterious channel. First, TRPM3 transcripts are alternatively spliced, leading to a bewildering number of splice variants, with significantly different functional properties

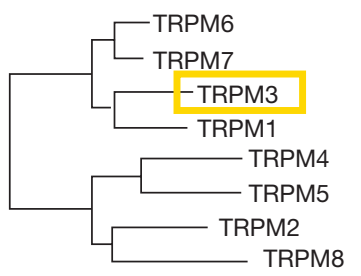

but unclear physiological relevance. In addition, the lack of a clear-cut activation mechanism has hampered detailed analysis of the biophysical properties and the functional role of the channel in its various splice isoforms. Although previous studies indicated that TRPM3 activity can be modulated by cell swelling, strong depolarization, removal of extracellular $\mathrm{Na}^{+}$and the endogenous ligand D-erythrosphingosine, none of these stimuli turned out to be robust or selective ${ }^{10}$.

b

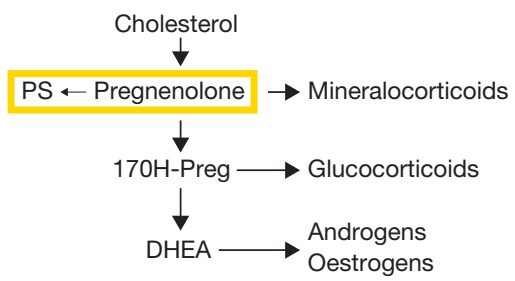

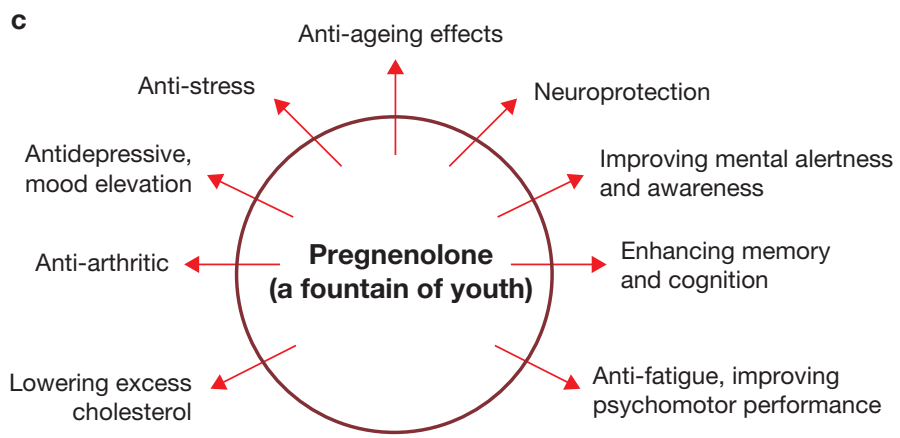

Figure $1 \mathrm{~A}$ 'marriage' between the transient receptor potential channel TRPM3 and the neurosteroid PS. (a) Phylogentic tree of the melastatin subfamily of TRP channels. Note that TRPM3 is the closest relative of the founding member melastatin (TRPM1). (b) Pregnenolone is synthesized from cholesterol and is considered to be the prohormone of all steroid hormones. $17 \alpha$-hydroxylase activity converts pregnenolone to 17 hydroxypregnenolone $(170 \mathrm{H}$-pregnenolone) and converts progesterone to $170 \mathrm{H}$-progesterone. DHEA (dehydroepiandrosterone) is converted to testosterone and oestrogenes. Sulphonation of pregnenolone leads to the formation of PS, a robust TRPM3 agonist. (c) Some of the positive effects attributed to pregnenolone, explaining its epithet 'fountain of youth'. 


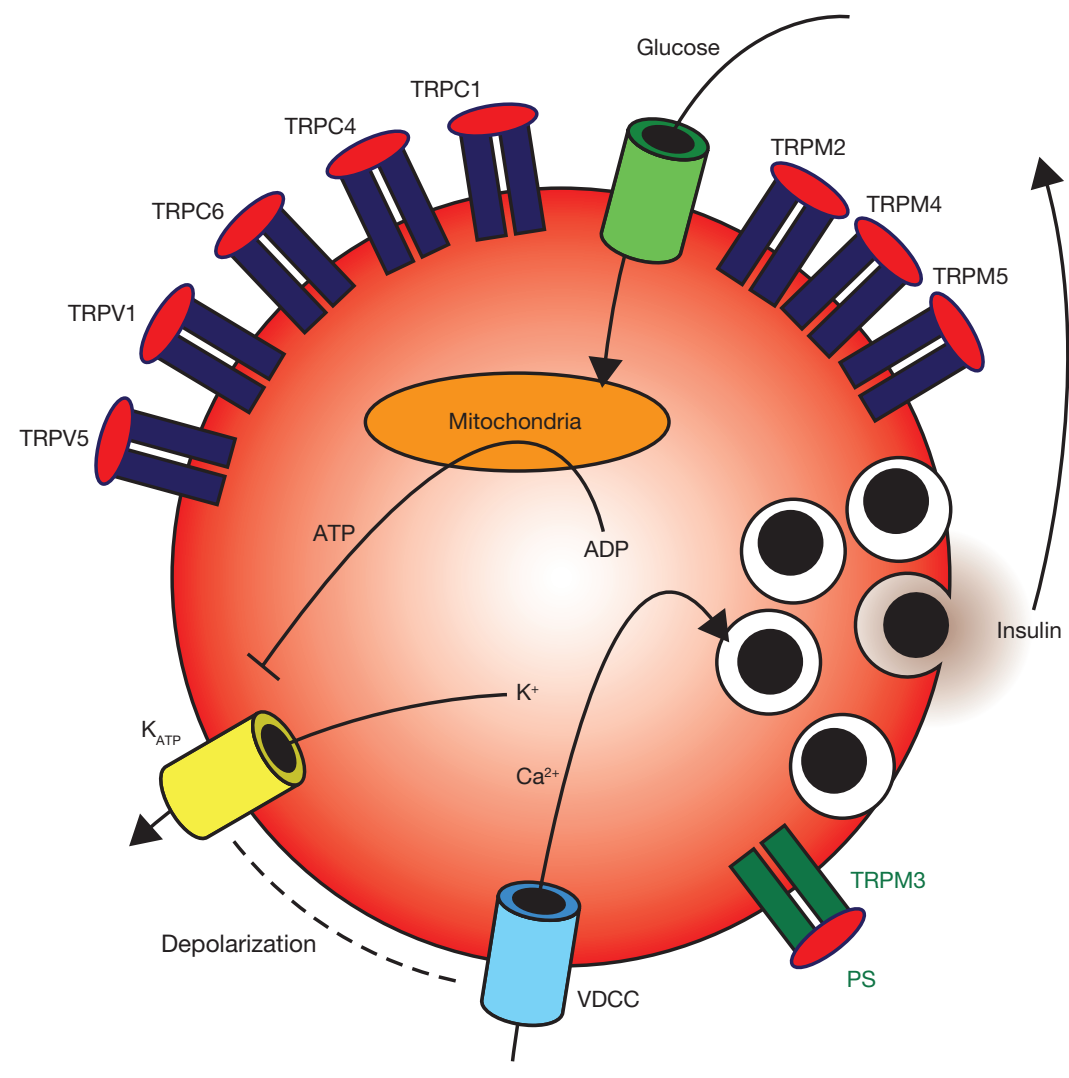

Figure 2 TRP channels in pancreatic $\beta$-cells. A schematic representation of a pancreatic $\beta$-cell: ATP production following glucose intake leads to inhibition of $\mathrm{K}_{\text {ATP }}$ channels, which results in cell depolarization, activation of voltage-activated L-type $\mathrm{Ca}_{\mathrm{v}}$ channels (VDCC) and finally, $\mathrm{Ca}^{2+}$ dependent exocytosis of insulin. At least nine different TRP channels are expressed in pancreatic $\beta$ cells; however their physiological role is not clear. Wagner et al. ${ }^{9}$ now show that activation of TRPM3 by PS evokes $\mathrm{Ca}^{2+}$-influx and promotes insulin release.

The study by Wagner et al. provides the first evidence for direct activation of a TRP channel by a steroid hormone. This is an important finding for a number of reasons. First, the effect of PS on TRPM3 is robust, producing an immediate and rapidly reversible effect, which makes it an excellent tool with which to study the properties of the channel, both in heterologous expression systems and in the cell types where TRPM3 expression has been reported. Therefore research on TRPM3 can now really begin, thanks to the discovery of a selective agonist. Second, another TRP channel has been 'deorphanized' by the identification of an endogenous agonist. In this respect, there are still many ligand-gated TRP channels for which an endogenous ligand is not known. Third, given that TRPM3 is expressed in several areas of the brain, these findings reveal a molecular player that may be involved in the various beneficial effects of PS on the brain. The study by Wagner et al., however did not investigate the role of TRPM3 as a neurosteroid receptor in the brain. Instead, this work linked
PS and TRPM3 to the release of insulin from pancreatic islets, an important endocrine function. It showed that TRPM3 protein is expressed in pancreatic $\beta$ cells, and elegantly illustrated that PS can augment glucose-induced insulin secretion from pancreatic islets. This raises the possibility that PS could be used to lower blood sugar in diabetic patients, and may be a new lead for anti-diabetic drug development. Moreover, it is interesting to speculate how PS might create crosstalk between insulin release and stimulation of brain functions that result in alleviating stress and fatigue, and ultimately improved mood, alertness and memory performance.

The connection between TRPM3 and pancreatic $\beta$ cells is particularly intriguing. Until recently, the $\mathrm{K}_{\mathrm{ATP}}$ (Kir6.2, SUR1) channel has been considered to be the master regulator of insulin release. Closure of $\mathrm{K}_{\text {ATP }}$ channels following glucose uptake and metabolism causes membrane depolarization, which then triggers $\mathrm{Ca}^{2+}$-dependent insulin exocytosis ${ }^{11}$. However, in the past two years, reports have appeared suggesting that the $\beta$ cell plasma membrane is packed with different TRP channels. Indeed, in addition to TRPM3, TRPC1, TRPC4, TRPC6, TRPV1, TRPV5, TRPM2, TRPM4 and TRPM5 have also been shown to be expressed in pancreatic $\beta$ cells ${ }^{12}$. Currently, the role of these TRP channels in $\beta$-cell (patho) physiology is a complex puzzle, but the use of selective agonists such as PS will be highly instrumental in solving it (Fig. 2).

To muddy the waters of the putative 'fountain of youth', this study does not provide a satisfactory answer to the question of whether PS is a physiologically relevant activator of TRPM3 channels. Indeed, the authors needed to use micromolar concentrations of PS for robust TRPM3 activation, and it is uncertain whether such high concentrations actually occur in vivo at the sites where TRPM3 is functionally expressed. Typical plasma PS concentrations are in the nanomolar range, and so far little is known about the free (as opposed to bound) PS concentration. Wagner et al. point out that PS concentrations may rise locally to micromolar concentrations, and that in certain conditions PS levels are significantly elevated, such as during mid-pregnancy ${ }^{13}$, in patients with 21-hydroxylase deficiency ${ }^{14}$, in women with anxiety-depressive disorders ${ }^{15}$ and in patients with hyperthyroidism ${ }^{16}$. It may be hypothesized that additional cellular factors might increase the sensitivity of TRPM3 for PS. Further research, for example using TRPM3-deficient mice, will be required to establish the role of TRPM3 as a bona fide steroid receptor.

1. Mayo, W., Le Moal, M. \& Abrous, D. N. Horm. Behav. 40, 215-217 (2001).

2. Vallee, M., Mayo, W. \& Le Moal, M. Brain Res. Brain Res. Rev. 37, 301-312 (2001).

3. Vallee, M., George, O., Vitiello, S., Le Moal, M. \& Mayo, W. Exp. Gerontol. 39, 1695-1704 (2004).

4. Flood, J. F., Morley, J. E. \& Roberts, E. Proc. Natl Acad. Sci. USA 92, 10806-10810 (1995).

5. Shen, W., Mennerick, S., Covey, D. F. \& Zorumski, C. F. J. Neurosci. 20, 3571-3579 (2000).

6. Eisenman, L. N., He, Y., Fields, C., Zorumski, C. F. \& Mennerick, S. J. Physiol. 550, 679-691 (2003).

7. Horak, M., Vlcek, K., Petrovic, M., Chodounska, H. \& Vyklicky, L., Jr. J. Neurosci. 24, 10318-10325 (2004).

8. Kudo, K., Tachikawa, E. \& Kashimoto, T. Eur. J. Pharmacol. 456, 19-27 (2002).

9 Wagner et al. Nature Cell Biol. 10, 1421-1430 (2008).

10. Oberwinkler, J. \& Phillipp, S. E. Handb. Exp. Pharmacol. 253-267 (2007).

11. Ashcroft, F. M. Nature 440, 440-447 (2006).

12. Nilius, B., Owsianik, G., Voets, T. \& Peters, J. A. Physiol. Rev. 87, 165-217 (2007)

13. Bicikova, M. et al. Steroids 67, 399-402 (2002).

14. de Peretti, E. et al. Acta Endocrinol. Suppl. 279, 259263 (1986).

15. Bicikova, M., Tallova, J., Hill, M., Krausova, Z. \& Hampl, R. Neurochem. Res. 25, 1623-1627 (2000).

16. Tagawa, N. et al. Clin. Chem. 46, 523-528 (2000). 